\title{
HOGARES UNIPERSONALES Y CURSO DE VIDA: DIVERSIFICACIÓN POR EDADES Y CONCENTRACIÓN ESPACIAL EN LAS REGIONES URBANAS DE MADRID Y BARCELONA
}

\author{
Cristina López Villanueva \\ Universidad de Barcelona \\ clopez@ub.edu \\ ORCID iD: https://orcid.org/0000-0002-7676-0765 \\ Isabel Pujadas Rúbies \\ Universidad de Barcelona \\ ipujadas@ub.edu \\ ORCID iD: https://orcid.org/0000-0001-7579-6603 \\ Miguel Rubiales Pérez \\ Universidad de Barcelona \\ mrubiales@ub.edu \\ ORCID iD: https://orcid.org/0000-0002-7364-2448
}

Recibido: 21/09/2017; Aceptado: 04/04/2019.

Cómo citar este artículo/Citation: López Villanueva, C.; Pujadas Rúbies, I. y Rubiales Pérez, M. (2019). Hogares unipersonales y curso de vida: diversificación por edades y concentración espacial en las regiones urbanas de Madrid y Barcelona, Estudios Geográficos, 80 (287), e012. https://doi.org/10.3989/estgeogr.201929.009

RESUMEN: Los hogares unipersonales en España se han ido transformando, aumentado su representatividad y diversificando su composición a la vez que han adquirido gran protagonismo en las áreas urbanas.

La distribución territorial de esta tipología de hogar no es homogénea en el interior de los espacios urbanos como tampoco lo son las características de las personas que viven solas. La especialización territorial de hogares unipersonales a nivel intrametropolitano es un fenómeno poco estudiado y el objetivo principal de análisis de este artículo. Las grandes ciudades, y en especial, los centros históricos son los que concentran una mayor proporción de hogares de una sola persona; no sólo debido a un mayor envejecimiento sino por ser localizaciones preferentes para la población joven y adulta que vive sola.

El artículo analiza la evolución de los hogares unipersonales en las dos mayores áreas urbanas de España (Madrid y Barcelona) poniendo de manifiesto las diferencias entre la capital y el resto de sus respectivas áreas examinando la localización territorial de los hogares de jóvenes (18-35 años), de adultos (36-64 años) y de mayores (> 65 años) a escala inframunicipal, a partir de la elaboración del cociente de localización, y explicando las características sociodemográficas de las personas que viven solas a partir de un análisis de árbol. El trabajo se nutre, principalmente, de los microdatos del Censo de 2011.

PALABRAS CLAVE: hogares unipersonales, distribución intrametropolitana, regiones metropolitanas, curso de vida, Madrid, Barcelona.

\section{SINGLE-PERSON HOUSEHOLDS AND LIFE COURSE: AGE DIVERSIFICATION AND SPACE CONCENTRATION IN THE URBAN REGIONS OF MADRID AND BARCELONA}

ABSTRACT: Single-person households have gained prominence and representativiness in urban areas. This growth has occurred along with the increase in the diversity of its composition.

The territorial distribution of this type of home is not homogeneous within urban spaces nor are the characteristics of people living alone. Territorial specialization of single-person households to intrametropolitan level is a little studied phenomenon and the main objective analysis of this article. The big cities, and especially, the historical centers are those that concentrate a greater proportion of single-person households; not only due to greater aging but because they are the preferred locations of other typologies of single-person households: the young and adult population living alone.

The article analyzes the evolution of single-person households in the two largest urban areas of Spain (Madrid and Barcelona) using 2011 census microdata; highlighting the differences between the capital and the rest of their respective areas by examining the territorial location of youth homes (18-35 years), of adults (36-64 years) and of elderly (> 65 years) at the inframunicipal level; analyzing the location quotients of key variables; applying a tree analysis to explaining the sociodemographic characteristics of people living alone.

KEY WORDS: single-person households, intrametropolitan distribution metropolitan regions, life course, Madrid, Barcelona. 


\section{INTRODUCCIÓN}

Los hogares unipersonales en España han aumentado en números absolutos y relativos, pasando de las 660.353 unidades de 1970 a las 4.193 .319 del año 2011. En 1970 representaban el 7,47\% del total de hogares donde residía el $2 \%$ de la población y en 2011 representan el 23,19\% donde vive el 9\% de la población española. Los hogares unipersonales fueron, durante décadas, responsables del crecimiento del número total de hogares. Junto a este incremento ha variado considerablemente su composición interna en el contexto de transformación de los hogares españoles.

Al menos dos han sido los determinantes del cambio en la estructura y composición, a los que se les une un tercero que tiene que ver con la localización de los hogares en el territorio urbano: a) en primer lugar, los cambios en las actitudes y comportamientos hacia formación y disolución de los hogares y las familias que se inscriben en el marco de la Segunda Transición Demográfica como ya pusieron de manifiesto los trabajos clásicos de: (Lesthaeghe, R., Van de Kaa, D., 1986), (Van de Kaa, D., 1987), (Surkyn, J., Lesthaeghe, R., 2004), Lesthaeghe, R. 2010) en segundo lugar, las estructuras demográficas existentes (Tomassini, C. et al., 2004), (Gaymu, J. et al., 2006), el envejecimiento de la población y la sobremortalidad masculina es un factor que incide en el hecho de residir solo a edades avanzadas después del fallecimiento del cónyuge o pareja; c) en tercer lugar, movilidad residencial que selecciona la población y también los hogares como ya han dado cuento de ello trabajos clásicos que relacionan la migración interna con diferentes hitos en el curso de vida (Clark, W. A., Onaka, J. L.,1983), (Tyrrell, N., Kraftl, P., 2015)

En primer lugar, la Segunda Transición Demográfica provee de un marco analítico -no exento de críticas en cuanto a su capacidad predictiva (Zaidi, B., Morgan, Ph., 2017) - para estudiar las profundas transformaciones que suceden, en un primer momento, en Europa Occidental desde la segunda mitad de los años sesenta. En los países del sur de Europa estos cambios presentaron particularidades propias y calendarios más tardíos (Naldini M., Jurado, T., 2013); en España no fueron visibles hasta mediados de los años ochenta después de la entrada en vigor de la Constitución en 1978 y de la reforma del Código civil en 1981. Las transformaciones, según el enunciado clásico, consisten en un decremento de la fecundidad y retraso a la edad media a la maternidad, un aumento considerable de los hijos nacidos de madres no casadas (Kohler,
H.P., Billari, F., Ortega, J.A., 2004), (Castro, T., MartínGarcía, T., 2013); aspecto al cual hoy se incorpora un incremento progresivo de la infecundidad (Esteve, A., Devolder, D., Domingo, A., 2016). La nupcialidad disminuye y la edad media al contraer matrimonio y formar pareja se retrasa (Billari, F., Castilglioni, M., Castro Martín, T., Michielin, F., Ongaro, F., 2002), (Muñoz-Pérez, F., Recaño, J., 2011); aumenta la cohabitación y se diversifican las formas de convivencia (Hiekel, N., Liefbroer, A., Poortman, A., 2014), (Domínguez Folgueras, M., Castro, T., 2013). Las tasas de separación y divorcio crecen (Perelli-Harris, B. et al., 2017). Estas transformaciones inciden en una flexibilización del curso de vida de los hogares. La ruptura de las uniones constituye una de las claves para generar itinerarios familiares reversibles aumentando el número de hogares más pequeños en tamaño y más diversos en composición, dando lugar a una disminución de hogares "tradicionales" formados por núcleos simples completos (parejas con hijos) y generando nuevas unidades unipersonales, sin núcleo o monoparentales (Castro, T., Seiz, M., 2014), (López Villanueva, C.; Pujadas, I., 2018).

Estas transformaciones contribuyen a una creciente complejidad en las transiciones a través del curso de vida y aumentando la probabilidad de los individuos de pasar alguna fase de su curso de vida residencial en un hogar unipersonal (Chandler, J. et al., 2004), (Jamieson, L., Simpson, R., 2013): al inicio de la vida adulta (Billari, F. C., Liefbroer, A.C., 2010), como consecuencia de una ruptura -separación o divorcio- (Demey, D. et al., 2013); entre la gente mayor (Zueras, P., Gamundi, P., 2013), o simplemente como una opción residencial más.

En segundo lugar, cabe tener en cuenta la estructura demográfica de la población, así pues las estructuras envejecidas tienden a generar una mayor proporción de hogares unipersonales. El aumento de la esperanza de vida y la mejora de las condiciones de salud y calidad de vida (Pérez, J., Abellán A., 2018) conllevan una mayor y más extensa independencia residencial a edades mayores, sobre todo en el caso de las mujeres debido su mayor supervivencia, ello explica el crecimiento de hogares unipersonales constituidos por mujeres viudas (López Villanueva, C., Pujadas, I., 2018).

En tercer lugar, debe considerarse la relación entre movilidad residencial y segregación espacial. Las dinámicas urbanas de desconcentración y suburbanización, impulsadas por la movilidad residencial (Clark, W. A., Onaka, J. L., 1983), (Kulu, H., Milewski, N., 2007), (Bonvalet, C., Bringé, A., Imbert, C., 2016) han contribuido a cambiar y redistribuir la estructura de 
la población y han seleccionado ésta en el territorio, así como la tipología de hogares reservando las grandes capitales para los hogares más pequeños y menos familiares, entre ellos los unipersonales, y el resto de las aglomeraciones urbanas para los hogares más familiares y numerosos en cuanto a dimensión media (Hall, R., Odgen, P., 2000; 2003). La movilidad residencial aparece ligada a los principales eventos relacionados con el curso de vida: emancipación, formación de una pareja, nacimiento de un hijo, separación o divorcio, jubilación o situación de dependencia (Tyrrell, N., Kraftl, P., 2015). Las dinámicas migratorias internas en las dos aglomeraciones urbanas estudiadas muestran saldos negativos en ambas capitales estudiadas, en todas las edades, aunque de manera más intensa en las franjas de edad joven (25-40 años) con destino a las periferias residenciales (Pujadas, I., Bayonai-Carrasco, J., 2015), (Garcia-Coll, A., López Villanueva, C., Pujadas, I., 2016). La saturación del mercado de la vivienda en las grandes ciudades centrales junto con la explosión de los precios al alza provocaron una migración de los demandantes de vivienda encabezada por las generaciones plenas de baby boommers (López Gay, A., 2018) que habían alcanzado la edad de la emancipación. Los flujos se dirigieron hacia destinos más alejados de los centros urbanos, más pequeños y con menores densidades, con una mejor relación precio-características de la vivienda (parque nuevo, mayor superficie, entorno natural, tranquilidad, etc.) que la burbuja inmobiliaria había construido (Garcia Coll, A., López Villanueva, C., 2018).

La movilidad residencial, como se ha referido, produjo un fuerte impacto sobre las estructuras por edades tanto de las grandes capitales como de sus áreas de expansión urbana dibujando una especialización territorial de estructuras más envejecidas en los centros y más jóvenes en las periferias, pero también incorporó un filtro sociodemográfico seleccionando la población que finalmente acabaría residiendo en la ciudad central (López Gay, A., 2011). El retorno de la población a los espacios centrales, vinculado a la especialización funcional del centro (Musterd, S., 2006) selecciona la población y también la tipología de los hogares; la potenciación de espacios centrales como centros de actividad económica atrae profesionales altamente cualificados, ello explica el crecimiento de hogares unipersonales de hombres y mujeres profesionales jóvenes en el centro de las grandes ciudades que se erigen como lugares atractivos para adultos y jóvenes con una expectativa de creciente movilidad social (Bonvalet, C.; Bringé, A., Imbert, C., 2016). Los cambios en la estructura y composición de los hogares se convierten, también, en agentes de transformación urbana y deben ser tenidos en cuenta en los trabajos sobre las tendencias y problemas urbanos (Buzar, S., Odgen, Ph. and Hall, R., 2005).

Pocos trabajos sobre las características y localización de los hogares unipersonales en las áreas urbanas a escala inframunicipal se han realizado en España. Con esta aportación se busca contribuir al análisis de la evolución de los hogares unipersonales en las dos mayores áreas metropolitanas de España (Madrid y Barcelona) poniendo de manifiesto las diferencias entre las capitales y el resto de la región y estudiando la localización territorial de los hogares unipersonales de jóvenes (18-35 años), adultos (36-64 años) y de mayores (> 65 años) a escala inframunicipal y explicando las características sociodemográficas de las personas que viven solas.

\section{FUENTES Y METODOLOGÍA}

En la primera parte del artículo se analiza la evolución de los hogares unipersonales en las ciudades de Madrid y Barcelona y en el resto de sus entornos metropolitanos, una primera cuestión es pues, delimitar las áreas urbanas. Mientras que Barcelona tiene bien delimitada una Región Metropolitana definida por el Plan Territorial General de Catalunya en 1995 y formada por 164 municipios, en Madrid no existe una delimitación "oficial". Con el objetivo de hacer comparables ambos espacios se han considerado para Barcelona su Región Metropolitana (RMB) con los 164 municipios antes referidos y para la de Madrid la Comunidad (CM), integrada por 178 municipios.

La fuente principal para el estudio de los hogares unipersonales han sido los microdatos del Censo de la Población Española de 2011 que se han complementado con los datos de los Censos de 2001 y de 1991.

La utilización de los microdatos ha permitido un mayor nivel de detalle en el estudio de los hogares aunque presenta algunas limitaciones: en primer lugar, no hay que olvidar que el Censo de 2011 no es de carácter universal, sino que toma forma de una gran encuesta y ello impone límites al nivel de desagregación territorial y al número de cruces entre las distintas variables; en segundo lugar, los datos se refieren a un momento concreto, la fecha censal, con lo cual se desconoce la duración del hecho de vivir en solitario $\mathrm{y}$; en tercer lugar, pueden existir declaraciones incorrectas (voluntarias o no) como las que se concentran en las zonas turísticas con elevados porcentajes de hogares unipersonales. 
Con la finalidad de dar cuenta de los objetivos que persigue el presente trabajo se ha elaborado la siguiente estrategia:

a) Para el análisis de la localización territorial de los hogares unipersonales a escala inframunicipal ha sido necesario realizar una serie ajustes metodológicos para conseguir una significación estadística, procediendo a agrupar secciones censales hasta conseguir unidades territoriales de más 20.000 habitantes -siguiendo las recomendaciones del INE-. Si bien es cierto que esta agrupación permite diferenciar los centros históricos de las ciudades de tamaño medio y grande respetando las delimitaciones administrativas preexistentes (municipios, distritos y/o barrios) obliga a agrupar municipios menores de 20.000 habitantes, resultando 212 unidades territoriales en la RMB y 249 en la CM.

b) Para el estudio de la concentración de los hogares unipersonales se ha construido un cociente de localización (QL) que relaciona la concentración relativa de los hogares de una sola persona en comparación con la media de la región metropolitana. Se han calculado tres cocientes de localización para los tres grandes grupos de edad (18-35); (36-64) y (>65). Junto con los resultados obtenidos, utilizando la desviación típica como medida de dispersión se han definido 5 categorías diferentes: subrepresentación absoluta; subrepresentación relativa; ligera concentración, concentración relativa y concentración alta. El QL se ha calculado según la siguiente relación

$$
Q L=\frac{X_{i} / T_{i}}{X / T}
$$

Donde $X_{i}$ significa la población que vive sola en la unidad espacial $i, T_{i}$ la población total de la misma unidad, y $X$ la población que vive sola en el conjunto de la región metropolitana y $\mathrm{T}$ la población total. Los valores por encima de 1 significan la existencia de concentración, mientras que por debajo de 1 indican una menor representación que en el conjunto estudiado.

c) Para el análisis de las características sociodemográficas de la población de los hogares unipersonales se ha realizado un análisis de árbol (Lind, D.A., Marchal, W.G., Wathen, S.A.,2012) de cuatro niveles. El objetivo es identificar las variables que marcan una mayor diferencia en los porcentajes de hogares unipersonales y seleccionar aquella que produce una diferencia mayor (a partir del contraste de Chi cuadrado). El análisis establece una primera división para cada gran grupo de edad: la población que reside en hogares unipersonales menores de 35 años, la que vive sola entre 36 y 64 y la mayor de 65 años. Con este método se generan subgrupos en función de las variables independientes elegidas como la edad, el sexo, la nacionalidad, el nivel de instrucción, la categoría profesional y el tamaño del municipio de residencia. La variable independiente que genera subgrupos con mayores diferencias porcentuales de observaciones (a partir del contraste de Chi cuadrado) es seleccionada en primer lugar. Este método se aplica recursivamente en cada nivel generando así una selección de las variables que mejor podrían explicar la propensión a formar hogares unipersonales y, podría ser la base de la elaboración de un modelo predictivo. Este análisis proporciona una selección estadísticamente significativa de las variables más determinantes, al tiempo que propone cierta jerarquización en su importancia.

\section{LOS HOGARES UNIPERSONALES COMO MOTOR DE CRECI- MIENTO DEL NÚMERO TOTAL DE HOGARES. MÁS HOGA- RES, MENOS POBLACIÓN}

Los hogares unipersonales en España han sido los protagonistas del crecimiento del número total de hogares españoles, aunque esta tendencia ha cambiado ligeramente en el último decenio y ello constituye una destacable novedad.

La población española creció de forma exigua y sostenida desde 1981 hasta el año 2000, momento a partir del cual la llegada de un importante contingente de población de nacionalidad extranjera contribuyó a un incremento sin precedentes. Esta aportación coincidió, necesariamente, con un período de extraordinaria intensidad en la formación de hogares; la primera fase de esta inmigración trajo consigo la constitución de hogares complejos (sin núcleo, extensos o de dos núcleos o más) (Domingo, A., Bayona, A., 2010) factor que unido a la crisis económica frenó el protagonismo del incremento de las unidades de una sola persona. Durante el decenio 2001-2011 fueron los hogares de dos personas los que mayor incremento presentaron.

\section{a) Crecimiento de los hogares y de la población}

La intensidad del crecimiento de hogares españoles ha sido superior a la de la población con lo cual, el tamaño medio de personas por hogar se reduce pasando de los 3,85 miembros de 1970 a los 2,86 de 2011. Las regiones metropolitanas de Madrid y Barcelona han seguido un proceso paralelo al del conjunto español, aunque con matices. 
En ambas áreas urbanas, los hogares crecen con más intensidad que la población. Este hecho es especialmente relevante en el caso de las dos ciudades centrales, las cuales han experimentado un crecimiento negativo de población durante el decenio 1991-2001 y, sin embargo, han visto aumentar el número de hogares. Así se constata la reducción del número medio de personas por hogar pasando de las 3,11 a las 2,42 personas en la ciudad Madrid y de las 2,87 a las 2,36 personas en Barcelona en contraste con el resto de sus respectivas regiones metropolitanas en las cuales el número medio de miembros por hogar es más elevado. (Tablas 1 y 2 ).

El segundo aspecto a destacar es la gran diferencia en la intensidad del crecimiento de los hogares en las ciudades de Madrid y Barcelona y el resto de sus regiones metropolitanas. Mientras que el municipio de Madrid experimentaba una tasa de crecimiento anual del $1,09 \%$ y del $2,03 \%$ en los períodos $1991-2001$ y $2001-$ 2011, el resto de la CM presentaba una intensidad mu- cho más elevada, 3,86\% y 3,77\%. Lo mismo sucedía en la ciudad de Barcelona en comparación con el resto de su región metropolitana, aunque presentando una tasa de crecimiento algo más modesta, $0,37 \%$ y $1,41 \%$ en la ciudad frente al $2,53 \%$ y $2,48 \%$ en el resto de la RMB. La mayor intensidad del crecimiento de los hogares en el resto de las regiones metropolitanas se explica, en parte, por el proceso de suburbanización. La movilidad residencial del centro a las periferias urbanas, de personas jóvenes que buscan entornos residenciales más ajustados a sus necesidades y preferencias económicas, familiares o ambientales implica un incremento en la formación de nuevos hogares sobre todo de tipo nuclear simple (López Villanueva, C., Pujadas, I., BayonaCarrasco, J., 2013). La mayor intensidad del crecimiento de los hogares y de la población del resto de la CM en relación con el resto de la RMB puede explicarse por la anticipación del proceso suburbanizador de la RMB (López Villanueva, C.; Pujadas, I., 2015).

TABLA 1

EVOLUCIÓN DE LOS HOGARES, HOGARES UNIPERSONALES, POBLACIÓN Y № MEDIO DE PERSONAS POR HOGAR. MADRID, BARCELONA, RESTO DE LA COMUNIDAD DE MADRID Y RESTO DE LA REGIÓN METROPOLITANA DE BARCELONA 1991, 2001 Y 2011

\begin{tabular}{|c|c|c|c|c|c|c|c|c|c|c|c|c|}
\hline & \multicolumn{3}{|c|}{ Madrid } & \multicolumn{3}{|c|}{ Barcelona } & \multicolumn{3}{|c|}{ Resto CM } & \multicolumn{3}{|c|}{ Resto RMB } \\
\hline & 1991 & 2001 & 2011 & 1991 & 2001 & 2011 & 1991 & 2001 & 2011 & 1991 & 2001 & 2011 \\
\hline Hogares & 969.518 & 1.080 .364 & 1.320 .530 & 573.056 & 594.452 & 684.080 & 543.191 & 793.428 & 1.148 .850 & 788.829 & 1.012 .921 & 1.293 .852 \\
\hline Hogares unipersonales & 161.526 & 257.772 & 379.830 & 103.939 & 155.463 & 198.050 & 40.159 & 105.450 & 201.745 & 80.309 & 171.100 & 267.090 \\
\hline Población & 3.010 .492 & 2.938 .723 & 3.186 .595 & 1.643 .542 & 1.503 .884 & 1.611 .013 & 1.937 .063 & 2.484 .661 & 3.200 .655 & 2.620 .880 & 2.886 .506 & 3.412 .148 \\
\hline$\%$ Hog. unipersonales & 16,66 & 23,86 & 28,76 & 18,14 & 26,15 & 28,95 & 7,39 & 13,29 & 17,56 & 10,18 & 16,89 & 20,64 \\
\hline \% Pob. Hog. unipesonales & 5,37 & 8,77 & 11,92 & 6,32 & 10,34 & 12,29 & 2,07 & 4,24 & 6,30 & 3,06 & 5,93 & 8,87 \\
\hline № medio personas hogar & 3,11 & 2,72 & 2,41 & 2,87 & 2,53 & 2,36 & 3,57 & 3,13 & 2,79 & 3,32 & 2,85 & 2,64 \\
\hline
\end{tabular}

Fuente: Elaboración propia a partir del censo de la población española, 1991, 2001 y 2011. INE

TABLA 2

TASA DE CRECIMIENTO ANUAL DE LOS HOGARES, HOGARES UNIPERSONALES, POBLACIÓN Y SU VARIACIÓN RELATIVA. MADRID, BARCELONA, RESTO DE LA COMUNIDAD DE MADRID Y RESTO DE LA REGIÓN METROPOLITANA DE BARCELONA 1991- 2001; 2001-2011 Y 1991-2011

\begin{tabular}{|c|c|c|c|c|c|c|c|c|c|c|c|c|}
\hline & \multicolumn{3}{|c|}{ Madrid } & \multicolumn{3}{|c|}{ Resto CM } & \multicolumn{3}{|c|}{ Barcelona } & \multicolumn{3}{|c|}{ Resto RMB } \\
\hline & 1991-2001 & $2001-2011$ & $1991-2011$ & 1991-2001 & 2001-2011 & $1991-2011$ & 1991-2001 & 2001-2011 & $1991-2011$ & 1991-2001 & $2001-2011$ & $1991-2011$ \\
\hline Hogares & 1,09 & 2,03 & 1,56 & 3,86 & 3,77 & 3,82 & 0,37 & 1,41 & 0,89 & 2,53 & 2,48 & 2,51 \\
\hline $\begin{array}{l}\text { Hogares } \\
\text { unipersonales }\end{array}$ & 4,79 & 3,95 & 4,37 & 10,14 & 6,70 & 8,41 & 4,11 & 2,45 & 3,28 & 7,86 & 4,55 & 6,19 \\
\hline Población & $-0,24$ & 0,81 & 0,28 & 2,52 & 2,56 & 2,54 & $-0,88$ & 0,69 & $-0,10$ & 0,97 & 0,42 & 0,70 \\
\hline $\begin{array}{l}\text { \% Hog. } \\
\text { unipersonales }\end{array}$ & 7,20 & 4,90 & 12,10 & 5,90 & 4,27 & 10,17 & 8,01 & 2,80 & 10,81 & 6,71 & 3,75 & 10,46 \\
\hline $\begin{array}{l}\text { \% Pob. Hog. } \\
\text { unipesonales }\end{array}$ & 3,41 & 3,15 & 6,55 & 2,17 & 2,06 & 4,23 & 4,01 & 1,96 & 5,97 & 2,86 & 2,94 & 5,81 \\
\hline $\begin{array}{l}\text { № medio } \\
\text { personas hogar }\end{array}$ & $-0,39$ & $-0,31$ & $-0,69$ & $-0,43$ & $-0,35$ & $-0,78$ & $-0,34$ & $-0,17$ & $-0,51$ & $-0,47$ & $-0,52$ & $-1,00$ \\
\hline
\end{tabular}

Fuente: Elaboración propia a partir del censo de la población española, 1991, 2001 y 2011. INE 


\section{b) Hogares según número de miembros}

Si se atiende al no de miembros por hogar, el más representado en España es el de dos personas, (con una proporción del 30,09\%), seguido de los hogares de un solo miembro $(23,19 \%)$; así sucede, también, en las ciudades de Madrid y Barcelona las cuales presentan valores similares en la proporción de los hogares de dos personas en Madrid (31,04\%) y algo superiores en Barcelona (33,64\%) y muy superiores en la proporción de hogares unipersonales $(28,76 \%$ y $28,95 \%)$ en Madrid y Barcelona, respectivamente. Cuando se comparan ambas ciudades con el resto de sus regiones metropolitanas se evidencia que el segundo lugar del ranking es ocupado por los hogares de tres personas, relegando a un tercer lugar los hogares unipersonales. Las tasas de crecimiento de los hogares según el $n$ o de personas (Figura 1) desvelan diferencias entre los centros urbanos y las periferias residenciales, así como entre Madrid y Barcelona. Mientras que en los restos metropoli- tanos crecen los hogares de uno a cuatro miembros durante el período 1991-2011 con una intensidad inversamente proporcional al no de miembros, perdiendo efectivos las unidades residenciales de cinco y más personas; en las capitales, sin embargo, tan solo crecen los hogares de una a tres personas en Madrid (con la excepción del período 2001-2011 donde las unidades de cuatro miembros se mantienen a un valor próximo a 0 , pero pierden el valor negativo) y de una y dos personas en Barcelona (con la misma excepción anterior aplicada a los hogares de tres miembros). Si bien el crecimiento de los hogares unipersonales protagonizó el incremento del total de los hogares, durante el período 2001-2011 en ambas ciudades, la intensidad del crecimiento de los hogares de una sola persona se frena y la tasa de crecimiento de los hogares de dos personas iguala (e incluso supera en el caso de la ciudad de Barcelona) la tasa de crecimiento de las unidades de un solo miembro.

FIGURA 1

TASA DE CRECIMIENTO ANUAL DE LOS HOGARES SEGÚN EL № DE PERSONAS. 1991-2001 Y 2001-2011. MADRID, RESTO DE LA CM, BARCELONA Y RESTO DE LA RMB
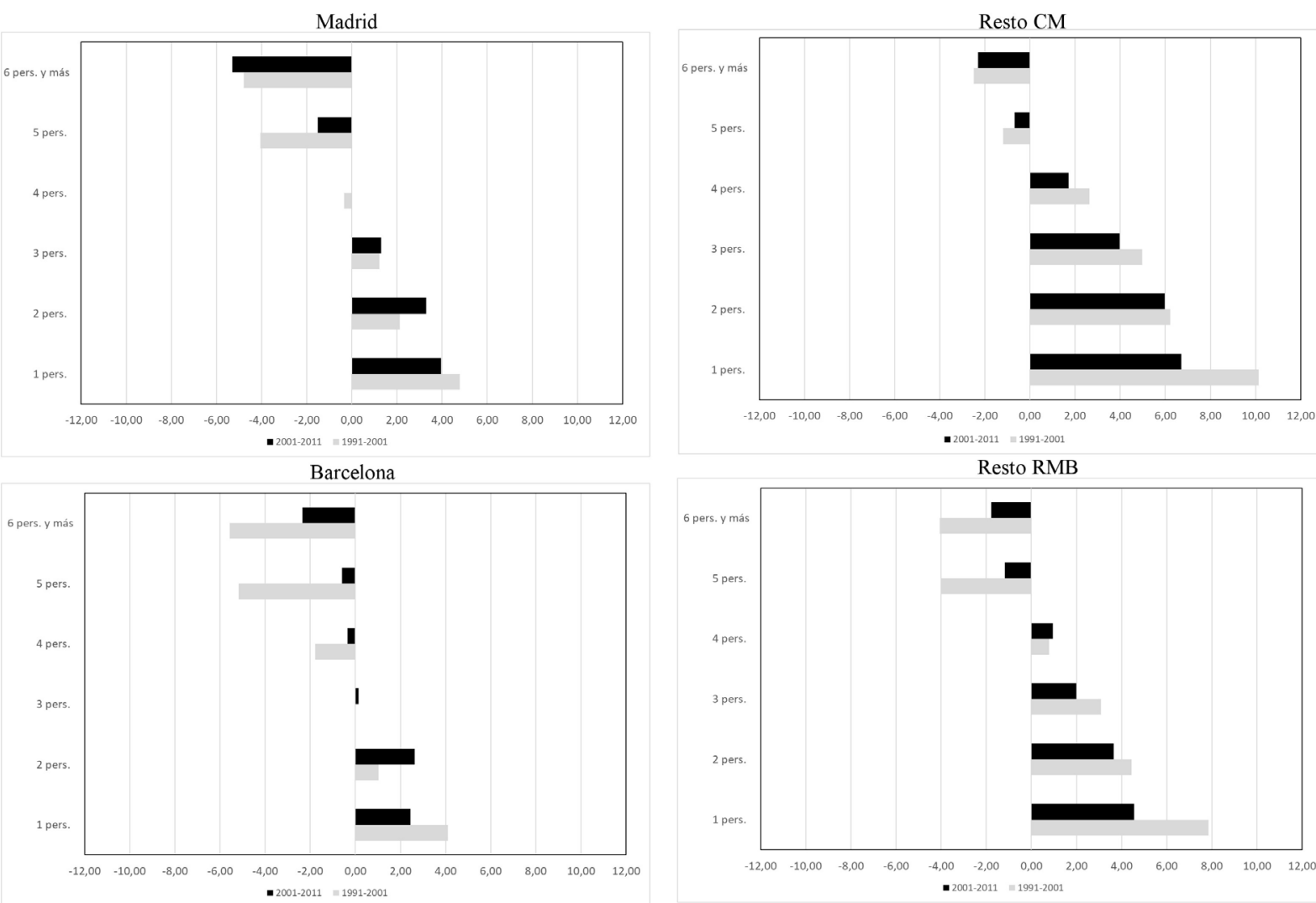

Resto RMB

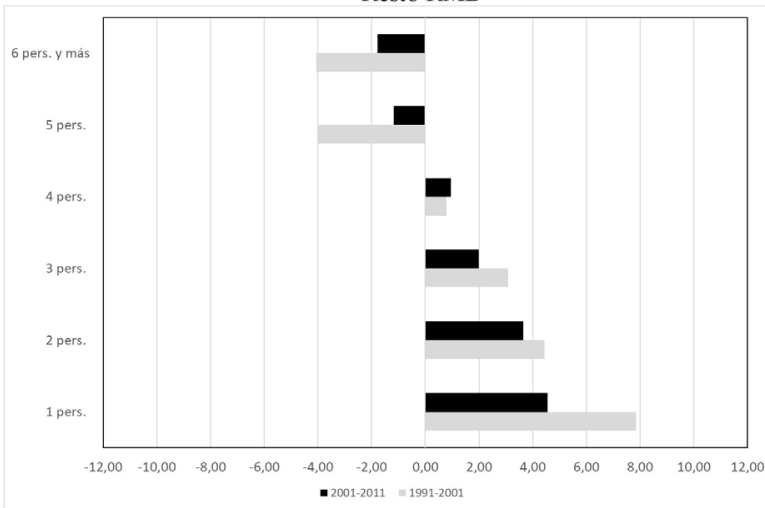

Fuente: Elaboración propia a partir del censo de la población española, 1991, 2001 y 2011. INE 


\section{c) Más hogares unipersonales en las áreas urbanas}

El tercer aspecto a señalar es la mayor representación de hogares unipersonales en las dos grandes capitales respecto al resto de sus áreas urbanas. Mientras que en 1991 la ciudad de Madrid y Barcelona tenían un $16,66 \%$ y $18,14 \%$ de hogares unipersonales donde residía el $5,37 \%$ y el $6,32 \%$ de la población, en el resto de la CM y de la RMB tan solo el 7,39\% y el $10,18 \%$ de los hogares estaban formados por una sola persona donde vivía el 2,07\% y el 3,06\% de la población. En 2011 la proporción de hogares unipersonales en ambos espacios urbanos ha aumentado considerablemente llegando a significar el $28,76 \%$ del total de los hogares en Madrid y el 28,95\% en Barcelona, en los cuales viven el $11,92 \%$ y el $12,29 \%$ de la población de ambas ciudades frente al $17,56 \%$ y $20,64 \%$ en el resto de sus regiones metropolitanas donde reside el 6,30\% y el $8,87 \%$ de la población, respectivamente. A pesar de que las grandes capitales continúan concentrando la residencia de personas que viven solas, la distancia entre la ciudad central y el resto de sus entornos metropolitanos tiende a reducirse, y éste es el cuarto aspecto a destacar.

En definitiva, disminuye el tamaño medio de los hogares, aumenta la proporción de los hogares unipersonales y éstos se localizan en las grandes ciudades y, como se describe más adelante, en los centros de las ciudades. Este modelo se ve alterado (Odgen, P.E., Schoebelen, F., 2005) por la extensión de los cambios de comportamientos demográficos y en las pautas en la formación y disolución de hogares más allá de las grandes ciudades, que conducen a un aumento de hogares unipersonales en el conjunto de ambas regiones urbanas (Hall, R., Odgen, P., 2004). La tasa de crecimiento anual r\% (Tabla 2) da cuenta de la mayor velocidad del crecimiento de los hogares unipersonales en el resto de las regiones metropolitanas que en las ciudades de Madrid y Barcelona.

\section{DiVERSIFICACIÓN DE LA ESTRUCTURA POR EDAD DE LA POBLACIÓN QUE VIVE EN HOGARES UNIPERSONALES. DIFERENCIA ENTRE CAPITAL Y REGIÓN METROPOLITANA}

Atendiendo a la proporción de personas que residen en hogares unipersonales según tres grandes franjas de edad $(<35 ; 36-64$ y $>65)$ sobre el total de población de dichos tramos etarios en tres momentos (1991, 2001 y 2011) (Tabla 3) se observa que:

En primer lugar, el incremento de los hogares unipersonales ha ido acompañado de una diversificación de sus características, aunque en ambas regiones urbanas la monoresidencialidad continúa teniendo un perfil envejecido y femenino.

En los tres momentos observados y tanto en Madrid como en Barcelona, así como en sus respectivas re-

TABLA 3

PROPORCIÓN DE PERSONAS QUE VIVEN EN HOGARES UNIPERSONALES SEGÚN GRANDES GRUPOS DE EDAD (<35; 36-64 Y >65) EN MADRID, BARCELONA, RESTO DE LA CM Y RESTO DE LA RMB. 1991, 2001, 2011

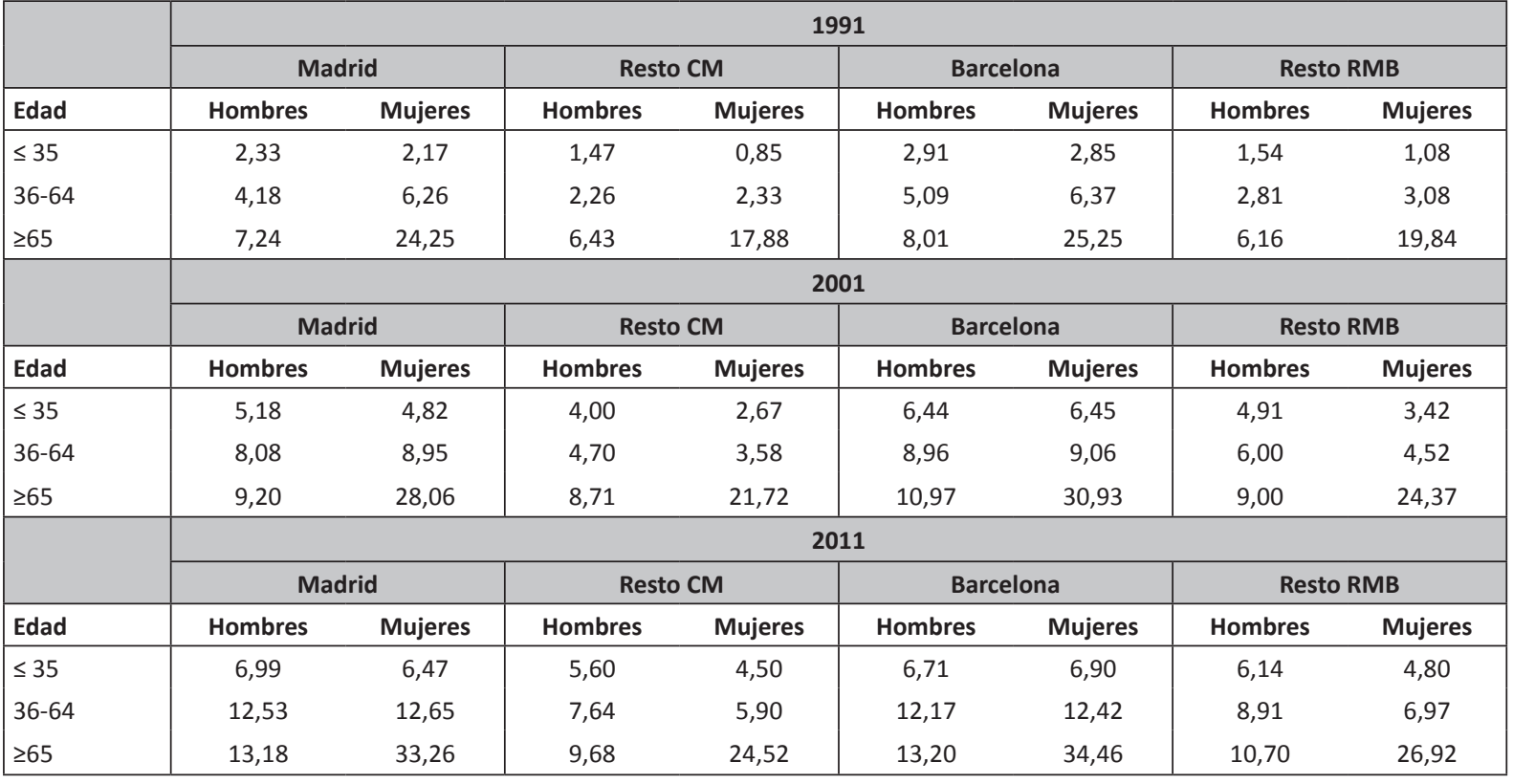

Fuente: Elaboración propia a partir del censo de la población española, 1991, 2001 y 2011. INE 
giones metropolitanas, destaca el absoluto predomino de las mujeres mayores de 65 años que residen en hogares unipersonales, pasando de representar una de cada cinco en la ciudad de Madrid y una de cada cuatro en el municipio Barcelona en 1991 a ser más de un tercio de la población en 2011 (Tabla 3). Las estructuras envejecidas y la mayor supervivencia femenina, así como la prolongación de la independencia residencial (López Doblas, J., Díaz Conde, M.P., 2013) explican esta composición en la que la viudez es el estado civil predominante (Pérez, J., Abellán, A., 2018).

Junto a este escenario cabe señalarse el extraordinario incremento de la proporción de población masculina, de 36 a 64 años residente en hogares unipersonales: en la ciudad de Madrid la proporción registrada en el año $2011(12,53 \%)$ se ha triplicado respecto a la de 1991 (4,18\%) y en Barcelona se ha multiplicado por 2,4 pasando de 5,09\% en 1991 a 12,17\% en 2011. El crecimiento de hombres de 36 a 64 años que viven en hogares unipersonales ha logrado igualar la proporción de mujeres que viven solas en el mismo intervalo etario en las dos grandes capitales y superarla en el caso de sus respectivas regiones metropolitanas. Son, pues, los hombres de esta edad los protagonistas del cambio en la composición de los hogares unipersonales. Varios trabajos ponen de manifiesto el incremento masculino de residir sin convivientes entre los 35 y 65 años (Jamieson, L., Wasoff, F., Simpson, R., 2009); (Demey, D., Berrrington, A., Evandrou, M., Falkingham, J., 2013). Los argumentos que se aportan apuntan a las situaciones de ruptura de parejas (cohabitantes o matrimonios) que generan hogares en transición compuestos por una sola persona y encabezados por hombres mientras que las mujeres lideran hogares monoparentales (Iacovou, M., Skew, A.J., 2011); estos trabajos evidencian, la importancia de los hombres que nunca han contraído matrimonio y no son separados o divorciados entre los que viven solos; en cambio constatan una incidencia mayor de la viudez entre las mujeres en estas edades.

La población de menos de 35 años es la que presenta una menor proporción de residencia en hogares formados por una sola persona; la emancipación tardía propia de las regiones del sur de Europa (Billari, F., Castilglioni, M., Castro Martín, T., Michielin, F., Ongaro, F., 2002); (Therborn, G., 2004) y los efectos de la crisis económica que dificultan todavía más el acceso a la vivienda, especialmente si éste se realiza en solitario (sin olvidar que esta etapa va unida a la formación de la pareja y la familia) explicarían la baja proporción de personas jóvenes residiendo en hogares unipersona- les. Pese a ello, en ambas capitales, la proporción de personas que viven en hogares formados por una sola persona menor de 35 años supera el 6,5\%, triplicando la proporción de 1991 en Madrid y duplicando la del mismo año en Barcelona. El cambio en esta franja de edad lo protagonizan las mujeres y no solo de las dos capitales sino del resto de las dos regiones metropolitanas estudiadas. Pese a la mayor representatividad de hombres jóvenes que residen sin convivientes, el crecimiento entre las mujeres es intenso llegando, en Barcelona, a igualar la proporción de varones.

2) En segundo lugar, y ésta es la tesis principal que sostiene el artículo, la proporción de personas que viven solas se concentra en las ciudades centrales, aunque este comportamiento va generalizándose hacia el resto de sus respectivas regiones metropolitanas.

En 2011 la ciudad de Madrid duplicaba el porcentaje de población residente en hogares de una sola persona, 11,92\%, frente al 6,30\% del resto de la CM; en Barcelona la proporción de personas que vivían solas era de $12,29 \%$ frente $8,87 \%$ del resto de la RMB. Trabajos clásicos como los llevados a cabo por el departamento de Geografía de la Universidad Queen Mary de Londres pusieron de manifiesto un patrón geográfico específico de los hogares unipersonales por edad (Hall, R., Odgen, Ph., Hill, C., 1997); (Hall, R., 2000, 2003 y 2004) mostrando una concentración de jóvenes o adultos que vivían solos en los centros de las grandes aglomeraciones urbanas (ligado, entre otras razones, al mercado de trabajo) mientras que los mayores se concentraban en espacios periféricos suburbanos. La especificidad de las ciudades de Madrid y Barcelona dibuja un escenario en el que conviven dos modelos: por un lado, las estructuras demográficas envejecidas con mayor propensión a formar hogares unipersonales (López Villanueva, C.; Pujadas, I., 2005; 2011); y por otro lado, la concentración de personas adultas y jóvenes que viven solas en las ciudades centrales las cuales ejercen una potente fuerza de atracción para los adultos jóvenes, dado su papel clave como centros de interacción cultural (Florida, R., 2002) y su potencial de movilidad social.

A pesar de este escenario descrito cabe destacar el importante crecimiento de hogares unipersonales, en todos los grupos de edad en el resto de las regiones metropolitanas de Madrid y Barcelona. En primer lugar, el mayor incremento en puntos porcentuales se ha experimentado en las mujeres mayores de 65 años; en 2011 una de cada cuatro mujeres mayores de 65 años vivían solas en el resto de la Región Metropolitana de Madrid y Barcelo- 
na. En segundo lugar, se triplican las poblaciones de hombres jóvenes y adultos que viven solos pasando a representar el 7,64\% del total de la población entre 36 y 64 años en el resto de la CM y el $8,91 \%$ en el resto de la RMB y, en tercer lugar, en términos relativos, se quintuplica la proporción de mujeres menores de 35 años que viven en hogares unipersonales pasando de ser prácticamente insignificante en 1991 superar el 4,50\% en 2011. Si bien es cierto que, tal y como se ha puesto de manifiesto, la concentración de hogares unipersonales se hace visible en las capitales de ambas aglomeraciones urbanas no hay que desestimar el crecimiento de hogares de una sola persona en el resto de las regiones metropolitanas de modo que los cambios en los comportamientos demográficos relativos a la formación y disolución de los hogares se extienden, rápidamente, a lo largo de todo el territorio metropolitano reduciendo los contrastes en la geografía de los hogares entre centros y periferias (Odgen, Ph., Schonoebelen, F., 2005).

3) En tercer lugar, existen algunos matices diferenciadores entre Madrid y Barcelona, aunque en 2011 las proporciones de personas que viven en hogares unipersonales en las ciudades de Madrid y Barcelona es muy similar, no ha sido igual la intensidad del cambio. Mientras que en la ciudad de Madrid el incremento de hogares unipersonales se ha producido durante el decenio 2001-2011 en Barcelona se produjo duran- te la década de los años noventa anticipándose a Madrid. Barcelona no solo se avanzó en la evolución de los comportamientos demográficos (López Villanueva, C., Pujadas, I., 2015) sino que su estructura demográfica más envejecida favoreció esta composición. Las proporciones de población que vive en hogares unipersonales son también superiores en el resto de la RMB que en la $\mathrm{CM}$, los procesos de suburbanización más tempranos en Barcelona que en Madrid (Pujadas, I., Bayona i Carrasco, J., 2015) puede ser un elemento que ayude a interpretar esta diferencia.

\section{ANÁLISIS TERRITORIAL DE LOS HOGARES UNIPERSONALES EN LAS REGIONES METROPOLITANAS DE MADRID Y BARCELONA}

La localización residencial de los hogares unipersonales a escala inframunicipal es el segundo objetivo de este estudio. Tal y como se ha referido se parte de la hipótesis de que las grandes ciudades y especialmente los centros históricos concentran mayor proporción de hogares unipersonales, no sólo debido al envejecimiento de la población sino, sobre todo, por ser los lugares preferentes para los jóvenes y adultos. El centro de la ciudad posee una mayor diversidad funcional, transporte y servicios públicos, un parque de viviendas de pequeño tamaño más aptas para el alquiler, y una vida cultural intensa que lo hace atractivo para los singles jóvenes y adultos. A ello hay que añadir la prolongación de la "juventud" a edades más maduras y las transiciones largas
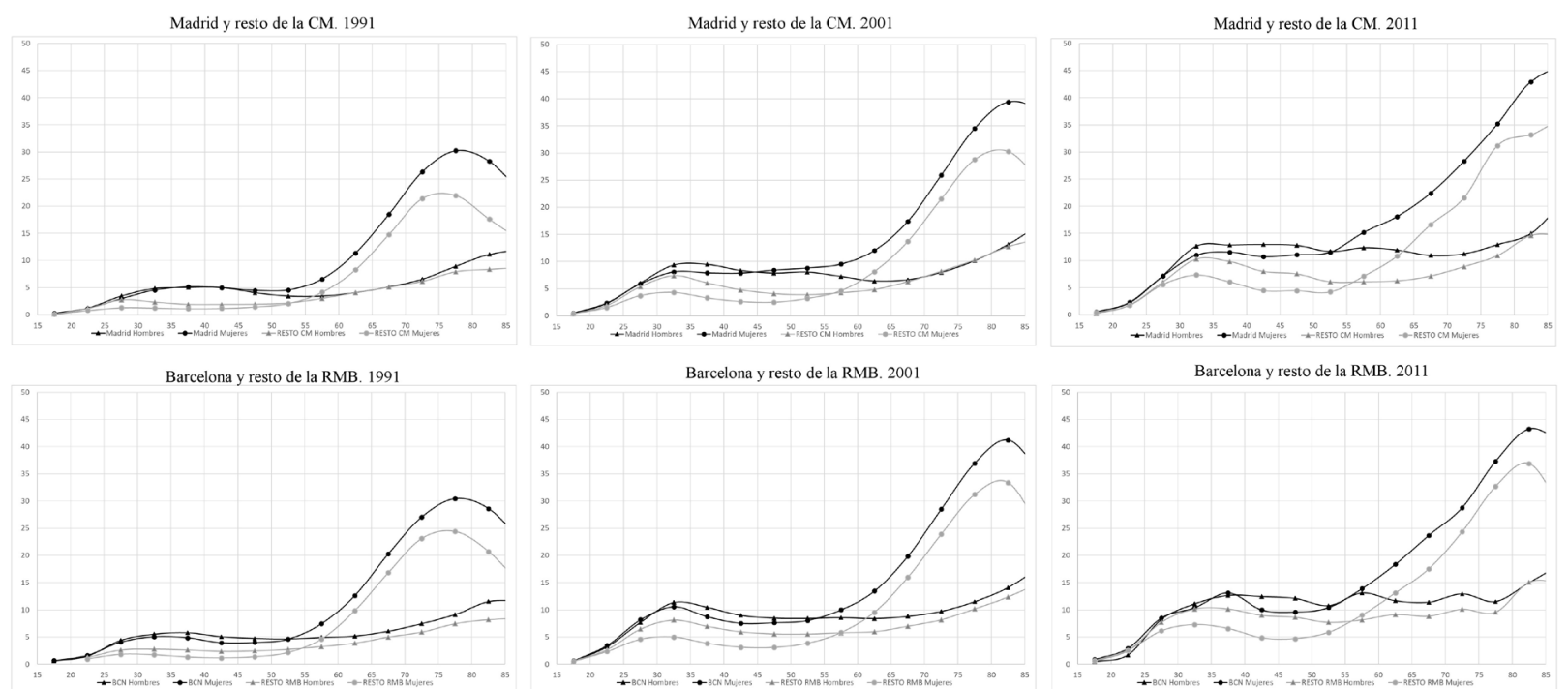

Fuente: Elaboración propia a partir del censo de la población española, 1991, 2001 y 2011. INE 
hacia la adultez (Rérat, P., 2012) que contribuyen a engrosar los hogares unipersonales de jóvenes en las grandes ciudades.

Para el análisis de la concentración de los hogares unipersonales se ha empleado el cociente de localización (QL) calculado para cada uno de los tres grandes grupos de edad.

Con los resultados alcanzados, y utilizando una medida de dispersión, la desviación típica, se han definido 5 categorías diferentes en función del nivel de concentración (Tabla 4). La primera indicaría la existencia de subrepresentación con valores por debajo de la media menos una desviación típica, las dos siguientes categorías, la subrepresentación relativa y la ligera concentración, indicarían valores alrededor de la media (entre más y menos una desviación típica), la cuarta denominada como de concentración relati$v a$ (entre una y dos desviaciones típicas por encima la media) y finalmente la quinta y última sería el umbral que utilizaríamos para delimitar la mayor concentración, concentración alta, con más de dos desviaciones típicas por encima de la media.
Los resultados (Tabla 4) muestran situaciones muy similares entre ambas regiones, aunque también algunas diferencias. Entre las similitudes se observa en ambas regiones urbanas una mayor dispersión de valores entre los jóvenes menores de 35 años, del mínimo de 0,276 al máximo de 2,898 en la RMB y entre 0,125 y 4,742 en la $\mathrm{CM}$ que traduce una mayor diferenciación espacial en la elección residencial de los solitarios más jóvenes, especialmente en la CM. Una dispersión que disminuye ligeramente en el grupo de adultos de 35-64 años, de un mínimo de 0,403 a 2,125 en la RMB y de 0,334 a 3,370 en la CM; de nuevo los valores más elevados de concentración de solos aparecen en la región metropolitana de Madrid. Por último, en el grupo de los mayores de 65 años, la diferenciación es menor en la RMB con un mínimo de 0,316 y un máximo de 1,963; en cambio en la región de Madrid el mínimo es inferior de 0,068 y el máximo de 2,534, muy superior al de la RMB, de modo que en Madrid se da una mayor concentración en algunos barrios de solitarios mayores. Así pues, las diferencias más significativas son la menor desviación estadística en la RMB entre sus unidades

TABLA 4

DISTRIBUCIÓN DE LOS COCIENTES DE LOCALIZACIÓN DE LOS HOGARES UNIPERSONALES POR NIVELES DE CONCENTRACIÓN EN LA CM Y LA RMB. 2011

\begin{tabular}{|c|c|c|c|}
\hline \multicolumn{4}{|c|}{ Comunidad de Madrid } \\
\hline Coeficiente de Localización & QL 18 a 35 años & QL 36 a 64 años & QL 65 años y más \\
\hline Subrepresentación (baja) & 0,125 a 0,310 & 0,334 a 0,518 & 0,068 a 0,628 \\
\hline Subrepresentación relativa (media - baja) & 0,311 a 1,000 & 0,519 a 1,010 & 0,629 a 1,021 \\
\hline Ligera concentración (media - baja) & 1,001 a 1,690 & 1,011 a 1,501 & 1,022 a 1,649 \\
\hline Concentración media & 1,691 a 2,380 & 1,502 a 2,484 & 1,650 a 2,278 \\
\hline Concentración alta & 2,381 a 4,742 & 2,485 a 3,370 & 2,279 a 2,534 \\
\hline Min & 0,125 & 0,334 & 0,068 \\
\hline Máx & 4,742 & 3,370 & 2,534 \\
\hline Media & 1,000 & 1,010 & 1,021 \\
\hline Desviación tipica & 0,689 & 0,491 & 0,628 \\
\hline \multicolumn{4}{|c|}{ Región Metropolitana de Barcelona } \\
\hline Coeficiente de Localización & QL 18 a 35 años & QL 36 a 64 años & QL 65 años y más \\
\hline Subrepresentación (baja) & $0,276-0,609$ & 0,403 a 0,664 & 0,316 a 0,607 \\
\hline Subrepresentación relativa (media - baja) & 0,610 a 1,002 & 0,665 a 0,980 & 0,608 a 0,977 \\
\hline Ligera concentración (media - baja) & 1,003 a 1,395 & 0,981 a 1,297 & 0,978 a 1,346 \\
\hline Concentración media & 1,396 a 1,788 & 1,298 a 1,614 & 1,347 a 1,716 \\
\hline Concentración alta & 1,789 a 2,898 & 1,615 a 2,125 & 1,717 a 1,963 \\
\hline Min & 0,276 & 0,403 & 0,316 \\
\hline Máx & 2,898 & 2,125 & 1,963 \\
\hline Media & 1,002 & 0,980 & 0,977 \\
\hline Desviación tipica & 0,393 & 0,317 & 0,370 \\
\hline
\end{tabular}


territoriales en todos los cocientes de localización, debido probablemente a un proceso más antiguo de metropolización y suburbanización que produce mayores similitudes entre centro y periferia (López Villanueva, C., Pujadas, I., 2015) contrariamente en la región de Madrid aparece mayor dispersión de valores en todos los grupos de edades, especialmente notorio entre el cociente de localización de los más jóvenes y los más mayores.

La representación de los cocientes de localización se muestra en las figuras 3, 4 y 5 . En estos mapas no se ha dibujado el valor del cociente en todas las unidades territoriales, sino únicamente sobre las áreas urbanas construidas, con lo que se obtiene una mayor precisión geográfica.

En Barcelona la concentración más alta de jóvenes sin convivientes aparece, en orden decreciente, en los barrios de la Barceloneta, en el barrio Gótico, la parte alta del Raval, en Gracia y en parte del Eixample y del Poble Nou, barrios todos ellos con viviendas de pequeño tamaño, una privilegiada situación central y en rápido proceso de renovación urbana y social, y en algunos casos de gentrificación acelerada (López Gay, A., 2018). El caso de la Barceloneta destaca por la propia tipología de las viviendas, los "quarts de casa" de reducido tamaño y por los procesos de renovación urbana que someten al barrio a una gentrificación en la que los jóvenes son unos de los agentes del cambio social.

En la Región metropolitana de Barcelona se distinguen igualmente los centros urbanos de las ciudades de mayor tamaño, como Terrassa, Mataró y Vilafranca del Penedés. La concentración media está más repartida por el territorio metropolitano, aunque aparecen algunas áreas de concentración especialmente en el litoral: en los municipios costeros del Baix Maresme, al norte y en la costa sur de Barcelona desde Gavá pasando por Catelldefels hasta Sitges.

También es visible en ciudades y capitales comarcales, como Sabadell, Granollers y Sant Cugat del Valles.

La baja concentración se da de forma dominante en las áreas de baja densidad de población y en los municipios más periféricos de la región metropolitana, zonas con crecimiento muy considerable de población y de viviendas unifamiliares en los últimos veinte años.

La distribución en Madrid presenta los mismos rasgos: gran concentración en los barrios centrales, Sol, Embajadores, Cortes, Justicia, Universidad, Chamberí y Salamanca, en gran parte de la almendra central, y menor concentración con el aumento de la distancia al centro. No obstante, conviene destacar algunas concentraciones significativas en la periferia sur y este de Madrid, en municipios con un pasado de gran crecimiento demográfico en los años sesenta y que actualmente pueden competir con el centro en oferta de vivienda de menor coste para la población joven, como es el caso de Alcorcón, Móstoles, Parla, Navalcarnero y Torrejón de Ardoz. Estos municipios comparten una gran heterogeneidad de cociente de localización, desde la alta concentración, como ya se ha citado, a concentraciones medias y muy bajas dibujando un perfil similar al de Madrid pero a una menor escala espacial, alta concentración en el centro y baja en su periferia municipal.

FIGURA 3

COCIENTE DE LOCALIZACIÓN DE LOS HOGARES UNIPERSONALES DE 18 A 35 AÑOS EN LA CM Y LA RMB, 2011
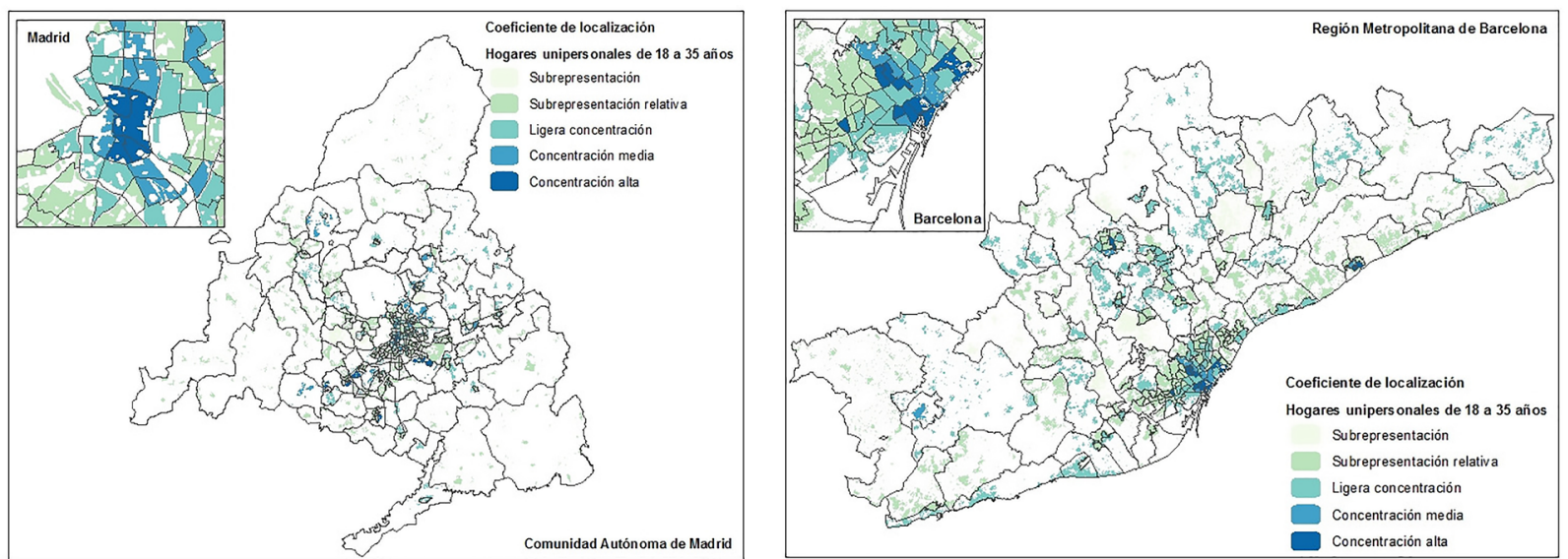

Fuente: Elaboración propia a partir de los microdatos del censo de la población española 2011. INE 
FIGURA 4

COCIENTE DE LOCALIZACIÓN DE LOS HOGARES UNIPERSONALES DE 36 A 64 AÑOS EN LA CM Y RMB, 2011
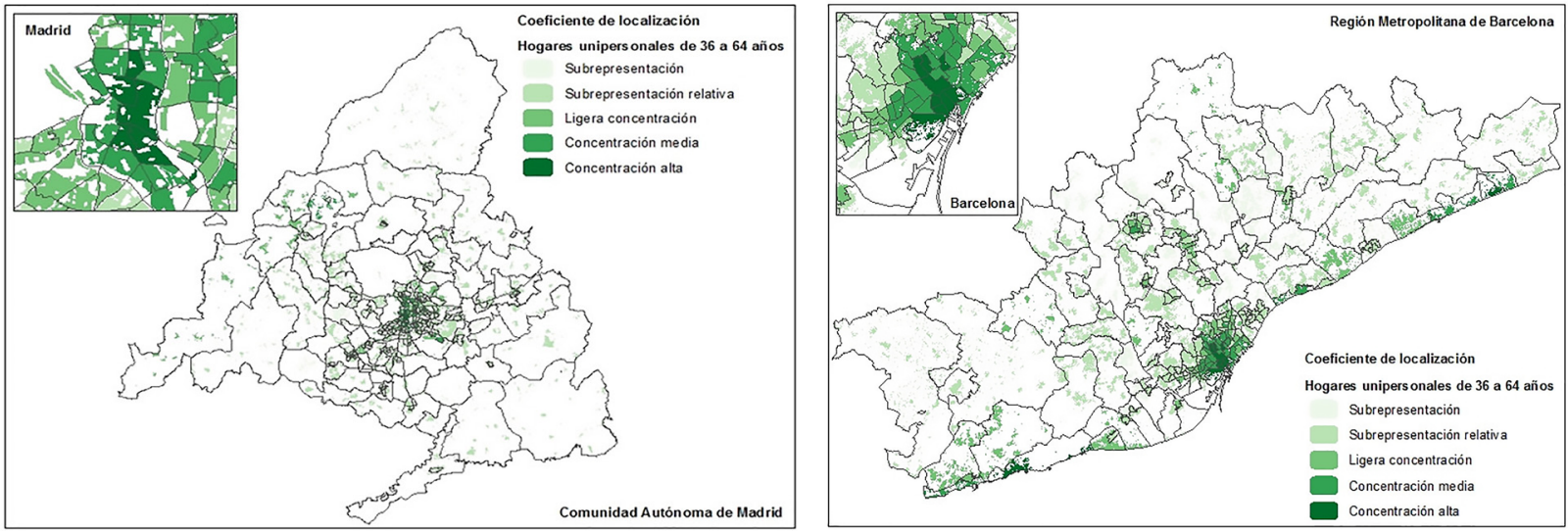

Fuente: Elaboración propia a partir de los microdatos del censo de la población española 2011. INE

La concentración espacial de los hogares unipersonales de la población adulta de 36 a 64 años sigue una pauta de mayor dispersión territorial. Así pues, en la $\mathrm{RMB}$, la suma de los dos grupos de mayor concentración, alta y media, de los jóvenes afecta a 20 unidades territoriales y en cambio, en el caso de los adultos abarca una mayor superficie urbana, en total 39 unidades espaciales por lo que su presencia está más repartida por el territorio. En la región de Madrid no ocurre lo mismo, la concentración alta y media de jóvenes afecta a 30 unidades y la de los adultos a 33. La distribución de los adultos que viven solos en Barcelona no diverge excesivamente de la que se ha referido en relación a los jóvenes: unos cocientes de concentración alta y media en los barrios centrales, Ciutat Vella, y Eixample; pero sí que aparecen algunas diferencias, con una gran presencia en el barrios del Eixample y en Gracia y contrariamente una menor representación en la Barceloneta y el Gòtic. En la RMB se repite su concentración en Terrasa y sobretodo en la línea litoral tanto en la comarca del Maresme, como en Castelldefels y Sitges. Esta localización costera puede dar lugar a algunos interrogantes, ¿̇son realmente hogares de solitarios, como opción residencial para la población divorciada que destina su segunda residencia a vivienda principal o el acceso a alquileres más bajos que en el centro de Barcelona, o bien se trata de empadronamientos atípicos con finalidades fiscales y que en realidad sean segundas residencias? Es posible que sea la suma de ambas posibilidades, y algunas más (Sabater, A., 2006).

En Madrid se da una concentración casi exclusiva de los cocientes más altos, concentración alta y media, en la misma capital, rellenando una parte importante de la almendra central. Sorprende su escasa concentración en los centros urbanos de las ciudades de tamaño grande.

FIGURA 5

COCIENTE DE LOCALIZACIÓN DE LOS HOGARES UNIPERSONALES MAYORES DE 65 AÑOS EN LA CM Y RMB, 2011
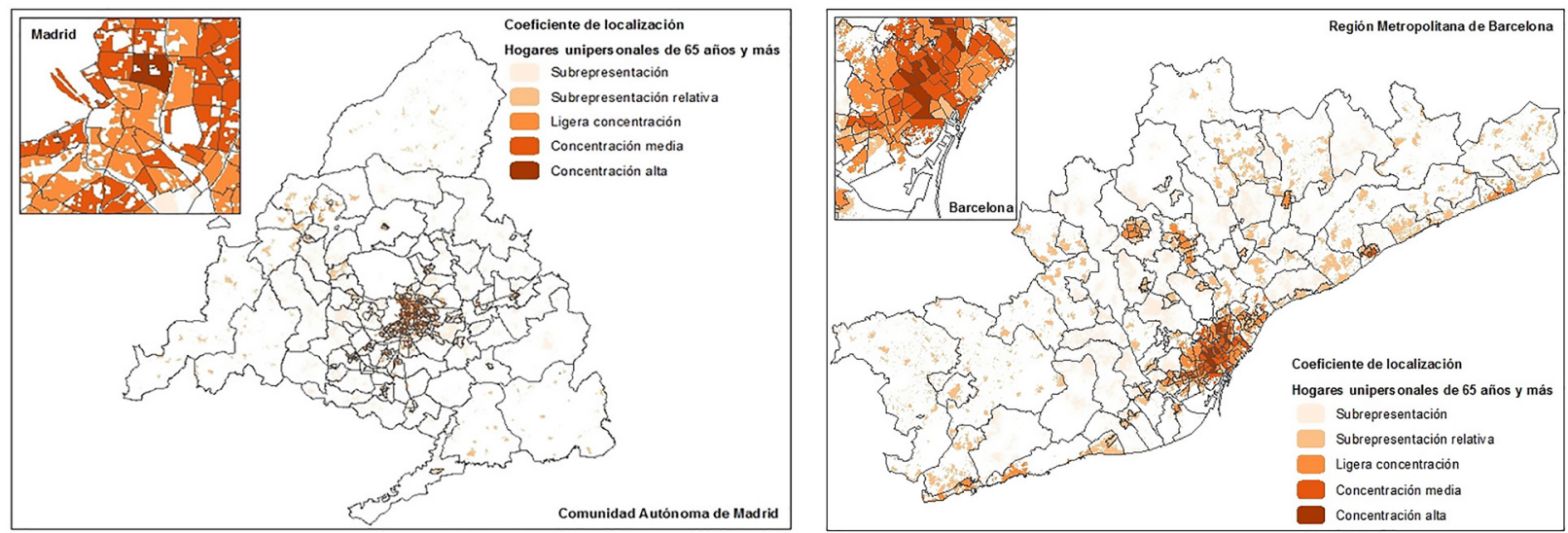

Fuente: Elaboración propia a partir de los microdatos del censo de la población española 2011. INE 
Las coronas metropolitanas son las que presentan una mayor subrepresentación de adultos solitarios, y en todas direcciones, al este en Alcalá de Henares, Coslada, y Rivas-Vaciamadrid, al oeste en Boadilla del Monte, Majadahonda y Pozuelo de Alarcón, al sur en los grandes municipios de Alcorcón, Fuenlabrada y Parla. Sería un ejemplo claro de anillos circuncéntricos de más a menos hogares unipersonales.

La localización de los hogares unipersonales de los mayores de 65 años sigue la pauta de la concentración alta en los centros urbanos, tanto en las dos grandes capitales, Madrid y Barcelona, como en el resto de ciudades de tamaño grande, especialmente en la RMB, como Terrassa, Sabadell y Mataró, mientras que en la región de Madrid no se da una alta concentración en los centros urbanos de las grandes ciudades de la periferia, sino una concentración ligera.

Madrid encabeza el ranking de una mayor extensión de barrios con concentración media y pocos con alta concentración, siempre situados en áreas próximas al centro, Chamberí, Salamanca, prolongándose hacia Ciudad Lineal. El centro histórico de Madrid no sobresale por ser una zona de alta concentración de solitarios mayores. Sin ninguna duda se está produciendo un proceso de substitución por residentes jóvenes y adultos fruto de un proceso gentrificador (Sorando, D., Ardura, A., 2018).

Es el mismo caso que se da en el centro histórico de Barcelona en rápido proceso de rejuvenecimiento de su población por el peso de la inmigración extranjera y por la entrada de jóvenes autóctonos en un proceso de substitución de los solitarios ancianos. Así pues la alta concentración no se da en el Raval, Gòtic o Barceloneta si no principalmente en gran parte del Eixample, especialmente el barrio de Sant Antoni, en Gracia, Sarrià y Sant Gervasi, barrios envejecidos con una estructura social de clases medias y altas. La concentración se desplaza también hacia barrios más populares del distrito de Nou Barris y en el Besós, barrios que crecieron con el peso de la inmigración en los años sesenta y ahora bastante envejecidos y con escasa renovación demográfica.

\section{ANÁLISIS DE LAS CARACTERÍSTICAS DE LOS HOGARES UNIPERSONALES EN LAS REGIONES METROPOLITANAS DE MADRID Y BARCELONA}

Este último apartado de análisis busca dar cuenta de la existencia de características sociodemográficas propias de aquellos que viven solos. Tras aplicar el correspondiente factor de elevación a los microdatos del
Censo de 2011, se obtiene una población de más de un millón de personas que viven solas, en total 1.088.190 habitantes que representan el 9,2\% de la población en Barcelona y Madrid. Esta población se ha analizado mediante un análisis de árbol que sirve para localizar aquellas variables que explican mejor la presencia o ausencia de hogares unipersonales (cuyos resultados se incluyen en el anexo $1.1 ; 1.2 ; 1.3$ ) y mediante un análisis descriptivo de las variables señaladas como relevantes tanto por el análisis de árbol como por la literatura existente sobre el tema.

En este caso, se explica la diversidad de los hogares unipersonales que previamente se han subdividido en tres grupos de edad, los menores de 35 años, los de 36 a 64 años y los de 65 y más, grupos que, como hipótesis de partida, se considera que responden a situaciones bien diferenciadas.

Para la población más joven, la primera subdivisión subraya la importancia de la categoría profesional, dividida en alta y baja, así la propensión a vivir sólo es considerablemente diferente: el $10,6 \%$ en la categoría alta contra el $1,9 \%$ en la baja. La segunda variable a destacar, y muy relacionada con la primera, es el nivel de formación que se ha dividido en dos grandes grupos, universitarios y no universitarios; para ambas categorías profesionales la proporción de solitarios siempre es mayor entre los universitarios, del $1,7 \%$ en los no universitarios al $6,5 \%$ de los universitarios en la categoría baja y paralelamente del $8,4 \%$ al $12,1 \%$ en la categoría alta. El perfil socioeconómico es la variable principal para explicar la mayor o menor proporción de hogares unipersonales entre los jóvenes, en cambio las variables sexo, nacionalidad y tamaño del municipio tienen un papel secundario.

Para la población adulta entre 36 y 64 años las variables independientes que actúan a favor de vivir en solitario son, en primer lugar, el nivel de formación, universitarios el $12,9 \%$ y no universitarios el $8,1 \%$; en segundo lugar la nacionalidad, española el 8,9\% y extranjera el 4,0 y finalmente el tamaño del municipio de residencia, con una menor diferenciación: $14,8 \%$ en las ciudades de más de 100.000 habitantes y el 10,4 en los municipios más pequeños y el 8,4 en los municipios intermedios entre 5.000 y 100.000 habitantes.

La primera subdivisión para la población mayor de 65 años es la variable sexo, así pues, el 30,6\% de las mujeres viven solas contra el $11,9 \%$ de los varones. La viudedad es el elemento diferencial que explica 
esta elevada desproporción, acompañada de una mayor capacidad organizativa entre la población femenina para su independencia residencial. En un segundo nivel aparece la dimensión territorial: las ciudades de mayor tamaño, más de 100.000 habitantes, tienen una mayor proporción de hogares unipersonales, sobre todo de mujeres, el $32,5 \%$ y mucho menos de varones el $12,4 \%$. El resto del territorio para ambas regiones metropolitanas es más homogéneo con pocas variaciones: en los municipios de menos de 5000 habitantes el $24,6 \%$ de las mujeres y el $11,9 \%$ de los hombres y en los municipios intermedios el $26,7 \%$ y el $10,3 \%$ respectivamente.

\section{CONCLUSIONES}

La distribución y concentración territorial en las regiones metropolitanas de Barcelona y Madrid de los hogares unipersonales se ha realizado a partir de un cociente de localización que ha permitido clasificar las unidades territoriales en áreas de subconcentración, concentración ligera y concentración media y alta, diferenciando a la vez los hogares unipersonales de jóvenes, de adultos y de mayores. El modelo dominante para los solitarios jóvenes es la ciudad central en Madrid y Barcelona, pero siempre vinculado a determinados barrios en fase de transformación sociodemográfica y renovación urbana, barrios donde la mezcla social es dominante y existen tendencias a la gentrificación. En las grandes ciudades de las periferias metropolitanas se repite la misma pauta de localización central. Contrariamente la subrepresentación se da en las áreas de urbanismo disperso más alejadas de los centros urbanos y con una tipología de nueva vivienda destinada a hogares familiares.

La concentración de los solitarios adultos es algo más extensa y afecta a territorios más diversos aunque con una especificidad, igualmente, de ciudad central. Además de esta zona central, los adultos forman hogares unipersonales en las áreas litorales en la región de Barcelona en las que domina un parque de viviendas secundarias, muy aptas para su uso como vivienda principal en caso de necesidad. Las razones de esta concentración litoral pueden estar relacionadas tanto con diferentes situaciones transitorias: mudanzas, reformas, primeros momentos tras de una separación o divorcio, como con el empadronamiento atípico en función de las necesidades fiscales. La subrepresentación, de nuevo, es dominante en las periferias suburbanas.

La concentración de hogares de mayores de 65 años está vinculada al proceso de envejecimiento, en general, y sobre todo su alta concentración en los centros urbanos. Barcelona y Madrid encabezan los mayores cocientes de concentración, seguidos de los centros urbanos del resto de ciudades. Envejecer en la propia vivienda ha sido la pauta a lo largo de las trayectorias vitales de la población española. De todos, la inmigración internacional y la movilidad interna de la población joven hacia determinados barrios centrales han modificado la concentración dominante de los mayores solitarios en algunas zonas centrales. En Barcelona ya no es Ciutat Vella donde se concentran las proporciones más altas de ancianos solitarios.

El análisis de árbol determina en primer lugar que el ciclo de vida, en función de tres grandes grupos de edad, es la variable que mejor explica la formación de hogares unipersonales. Estas diferencias no son solo cuantitativas. Los hogares unipersonales tienen, en cada momento del ciclo de vida, un significado y características diferentes que emerge cuando se subdivide en función de la siguiente variable:

Para los jóvenes menores de 35 años, la posibilidad de emanciparse sin un proyecto de convivencia en pareja aparece muy relacionada con la capacidad económica. Así los porcentajes de jóvenes que viven solos son sistemáticamente más altos para aquellos ocupados en las categorías profesionales más altas y entre aquellos con formación universitaria o superior.

Para los adultos entre 36 y 64 años, el mayor diferencial en la proporción de personas que viven solas, responde a un patrón territorial: si se localizan en los centros metropolitanos, o en las periferias. Es posible que la relación entre localización periférica y baja proporción de hogares unipersonales se encuentre mediada por la mayor facilidad para formar una familia con hijos fuera de los centros densos.

Finalmente, para las personas mayores de 65 años, la variable que marca mayores diferencias es el sexo, concretamente, la mayor esperanza de vida de las mujeres que hace les resulte más frecuente experimentar la viudez.

En los siguientes niveles ( 3 y 4 ) de subdivisión de los grupos, van apareciendo otras variables asociadas al estatus socioeconómico (los estudios universitarios y haber nacido en países del sur global) y a elementos residenciales (como el régimen de tenencia y la localización). En función del nivel socioeconómico, la "unipersonalidad" se asocia a la mayor capacidad: las personas universitarias, con nivel socioeconómico por 
encima de la media, viven solas en una mayor proporción; mientras que resulta menos frecuente que las personas nacidas en países de menor PIB per cápita (que presentan, de media, un menor nivel socio económico) vivan solas. El régimen de acceso a la vivien$\mathrm{da}$, si es mediante propiedad o alquiler, se asocia a la estabilidad o temporalidad. Cada vez que los niveles se subdividen en función de si la vivienda es alquilada o no, los mayores niveles de "unipersonalidad"

\section{BiBLIOGRAFÍA}

Billari, F. C., Liefbroer, A. C. (2010). Towards a new pattern of transition to adulthood? Advances in Life Course Research, 15(2-3), 59-75.

Billari, F., Castilglioni, M., Castro Martín, T., Michielin, F., Ongaro, F. (2002). Household and union formation in a Mediterranean fashion: Italy and Spain. En E. Klijzing, M. Corijn (Eds.), Dynamics of fertility and partnership in Europe. Insights and lessons from comparative research (Vol. II, pp. 17-41). New York, USA: United Nations.

Bonvalet, C., Bringé, A., Imbert, C. (2016). Urban dynamics and residential trajectories in Paris. Portuguese Journal of Social Science, 15(1), 25-46.

Buzar, S., Odgen, Ph. and Hall, R. (2005). Households matter: the quiet demography of urban transformation. Progress in Human Geography, 29(4), 413-436.

Castro, T., Martín-García, T. (2013). Fecundidad bajo mínimos en España: pocos hijos, a edades tardías y por debajo de las aspiraciones reproductives. En Esping-Andersen, G. (Coord.), El déficit de la natalidad en Europa: la singularidad del caso español (pp. 48-88). Barcelona, España: Obra Social La Caixa.

Castro, T., Seiz, M. (2014). La transformación de las familias en España desde una perspectiva sociodemogràfica. VII. Informe sobre exclusión y desarrollo social en España 2014. Informe Foessa.

Chandler, J., Williams, M., Maconachie, M., Collett, T., Dodgeon, B. (2004). Living alone: its place in household formation and change. Sociological Research Online, 9(3), 1-13.

Clark, W., Onaka, J. L. (1983). Life cycle and housing adjustment as explanations of residential mobility. Urban studies, 20(1), 47-57.

Demey, D. Berrrington, A. Evandrou, M. Falkingham, J. (2013). Pathways into living alone in mid-life: aparecen en las personas que se encuentran bajo un régimen de alquiler. Finalmente, aunque en niveles inferiores, también aparece la vinculación entre centralidad metropolitana y la vida en solitario en las subdivisiones de los mayores de 65 años.

Este ensayo estadístico ha aportado algunas conclusiones significativas, pero sobre todo ha abierto nuevas perspectivas de análisis para posteriores estudios.

Diversity and policy implications. Advances in Life Course Reserarch, 18(3), 161-174.

Domingo, A., Bayona, J. (2010). Los hogares de la población de nacionalidad extranjera en España en el año 2001. Papers, 95(3), 731-754.

Domínguez Folgueras, M., Castro, T. (2013). Cohabitation in Spain: No longer a marginal path to family formation. Journal of Marriage and Family, 75(2), 422-437.

Esteve, A., Devolder, D., Domingo, A. (2016). La infecundidad en España: tic-tac, tic-tac, tic-tac. Perspectives Demogràfiques, 1(1), 1-4.

Florida, R. (2002). The rise of the creative class: and how it's transforming work, leisure, community and everyday life. New York: Basic Books.

Garcia-Coll, A., López Villanueva, C., Pujadas, I. (2016). Movilidad residencial en tiempos de crisis. El caso de la Región Metropolitana de Barcelona. Scripta Nova, XX(549-4).

Garcia-Coll, A., López-Villanueva, C. (2018). The Impact of Economic Crisis in Areas of Sprawl in Spanish Cities. Urban Science, 2(4), 113. https://doi. org/10.3390/urbansci2040113

Gaymu, J., Delbès, C., Springer, S., Binet, A., Désesquelles, A., Kalogirou, S., Ziegler, U. (2006). Determinants of the living arrangements of older people in Europe. European Journal of Population/Revue européenne de Démographie, 22(3), 241-262.

Hall, R., Odgen, P. (2000). Households, reurbanisation and the rise of living alone in the principal french cities. 1975-1990. Urban Studies, 37(2), 367-390.

Hall, R., Odgen, P. (2003). The rise of living alone in inner London: Trends among the population of working age. Environment and Planning, 35(5), 871-888. 
Hall, R., Odgen, P. (2004). The second demographic transition, new household forms and the urban population of France during the 1990s. Transactions of the British Institute Geographers, 29(1), 88-105.

Hall, R., Ogden, P. E., Hill, C. (1997). The pattern and structure of one-person households in England and Wales and France. Population, Space and Place, 3(2), 161-181.

Hiekel, N., Liefbroer, A. C., \& Poortman, A. R. (2014). Understanding diversity in the meaning of cohabitation across Europe. European Journal of Population, 30(4), 391-410.

lacovou, M., Skew, A. J. (2011). Household composition across the new Europe: Where do the new Member States fit in? Demographic research, 25, 465-490.

Jamieson, L. and Simpson, R. (2013).Living Alone: Globalization, Identity and Belonging. Basingstoke: Palgrave Macmillan.

Jamieson, L., Wasoff, F., Simpson, R. (2009). Solo-living, demographic and family change: The need to know more about men. Sociological Research Online, 14(2), 1-16.

Kohler, H.P., Billari, F., Ortega, J.A. (2004). The emergence of Lowest-low fertility en Europe during the 1990's. Population and Development Review, 28(4), 641-680.

Kulu, H., Milewski, N. (2007). Family change and migration in the life course: An introduction. Demographic Research, 17, 567-590.

Lesthaeghe, R. (2010). The unfolding story of the second demographic transition. Population and development review, 36(2), 211-251.

Lesthaeghe, R., Van de Kaa, D. J. (1986). Twee demografische transities. Bevolking: groei en krimp, special issue of Mens en Maatschappij. Deventer: Van Loghum Slaterus, 9-24.

Lind, D.A, Marchal, W.G., Wathen, S.A., (2012). Estadística aplicada a los negocios y la economía. México D.F.: MCGraw Hill.

López Doblas, J., Díaz Conde, M. P. (2013). La modernización social de la vejez en España. Revista Internacional de Sociología, 71(1), 65-89.

López Gay, A. (2018). Cambio en la composición social y gentrificación en Barcelona: una mirada a través de los flujos migratorios residenciales. Revista $\mathrm{Pa}$ pers, 60, 80-93.
López Gay, A. (2011). ¿Vuelve el centro? Caracterización demogràfica de los procesos de reurbanización en las metrópolis españolas. En Pujadas, l. et al. (Eds.). Población y espacios urbanos (pp.163180). Barcelona, España: Dep. De Geografia Humana UB y $A G E$.

López Villanueva, C., Pujadas, I. (2005). Hogares y cambios residenciales. La diferenciación espacial de los hogares en la Región Metropolitana de Barcelona. Cuadernos Geográficos, 36, 409-435.

López Villanueva, C., Pujadas, I. (2011). Transformaciones sociodemográficas y territoriales de los hogares unipersonales en España. Boletín de la AGE, 05(55), 153-182.

López Villanueva, C., Pujadas, I. (2015). Transformaciones espaciales y demográficas en las regiones metropolitanas de Madrid y Barcelona. En Domínguez, M., López Villanueva, C (Coords.). Barcelona y Madrid. Procesos urbanos y dinámicas sociales (pp. 71-106). Madrid, España: Ed. Síntesis.

López Villanueva, C., Pujadas, I. (2018). Vivir solo en España. Evolución y características de los hogares unipersonales de la vejez. Panorama social, 28, 93-114.

López Villanueva, C., Pujadas, I., Bayona-Carrasco, J. (2013). Households Within The Residential Mobility Process: The Case Of The Barcelona Metropolitan Region. Archivio di Studi Urbani e Regionali, $108,57-84$

Muñoz-Pérez, F., Recaño, J. (2011). A century of nuptiality in Spain, 1900-2007. European Journal of Population, 27(4), 487-515.

Musterd, S. (2006). Segregation, urban space and resurgent city. Urban Studies, 43(8), 1325-1340.

Naldini, M., Jurado, T. (2013). Family and welfare state reorientation in spain and italy from a European perspective. Population Review, 52(1), 43-61.

Odgen, Ph, Schnoebelen, F. (2005). The rise of de small household: Demographic change and Household Structure in Paris. Population Space and Place, 11, 251-268.

Perelli-Harris, B., Berrington, A., Gassen, N. S., Galezewska, P., Holland, J. A. (2017). The rise in divorce and cohabitation: Is there a link? Population and development review, 43(2), 303-329.

Pérez, J., Abellán, A. (2018). Envejecimiento demográfico y vejez en España. Panorama Social, 28, 11-47. 
Pujadas, I., Bayona i Carrasco, J. (2015). Las migraciones residenciales en las regiones metropolitanas de Madrid y Barcelona. En Domínguez, M., López Villanueva, C. (Eds.), Barcelona y Madrid. Procesos urbanos y dinámicas sociales (pp. 43-69). Madrid. España: Ed. Síntesis.

Rérat, P. (2012). The new demographic growth of cities: the case of reurbanisation in Switzerland. Urban Studies, 49(5), 1107-1125.

Sabater,A. (2006). Una aproximación a los empadronamientos atípicos en Cataluña: cómputo y características sociodemográficas de un fenómeno generalizado. Documents d'Anàlisi Geogràfica, 46, 63-79.

Sorando, D., Ardura, A, (2018). Procesos y dinámicas de gentrificación en las ciudades españolas. $P a-$ pers, 60, 34-47.

Surkyn, J., Lesthaeghe, R. (2004). Value orientations and the second demographic transition (SDT) in Northern, Western and Southern Europe. Demographic research, 3, 45-86.
Therborn, G. (2004): Between sex and power: Family in the world 1900-2000. Routledge.

Tomassini, C., Glaser, K., Wolf, D. A., van Groenou, M. B., \& Grundy, E. (2004). Living arrangements among older people: an overview of trends in Europe and the USA. Population trends London,115, 24-35.

Tyrrell, N. and Kraftl, P. (2015). Lifecourse and Internal Migration. En Smith, D. et al (Eds.), Internal Migration. Geographical Perspectives and Processes (pp. 15-30). London: Ashgate.

Van de Kaa, D. (1987). Europe's second demographic transition. Population Bulletin, 42, 5-37.

Zaidi, B., Morgan, S. P. (2017). The second demographic transition theory: A review and appraisal. Annual review of sociology, 43, 473-492.

Zueras, P., \& Gamundi, P. M. (2013). Mayores que viven solos: una panorámica a partir de los censos de 1991 y 2001. Revista Española de Investigaciones Sociológicas (REIS), 144(1), 139-152. 
ANEXO 1.1

ANÁLISIS ÁRBOL. HOGARES UNIPERSONALES JÓVENES < 35 AÑOS

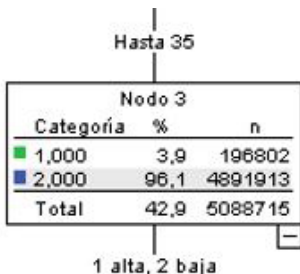

Valor $\mathrm{P}$ cortregido=0,000, Chi.

cuadrado $=177732,964, d f=1$

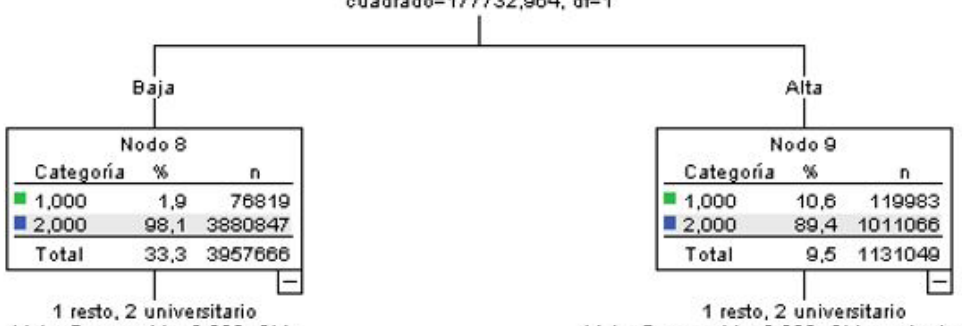

1 resto. 2 universitario

Valor $P$ corregido $=0,000$, Chi

1

cuadrado $=26578,862, \mathrm{dt}=1$
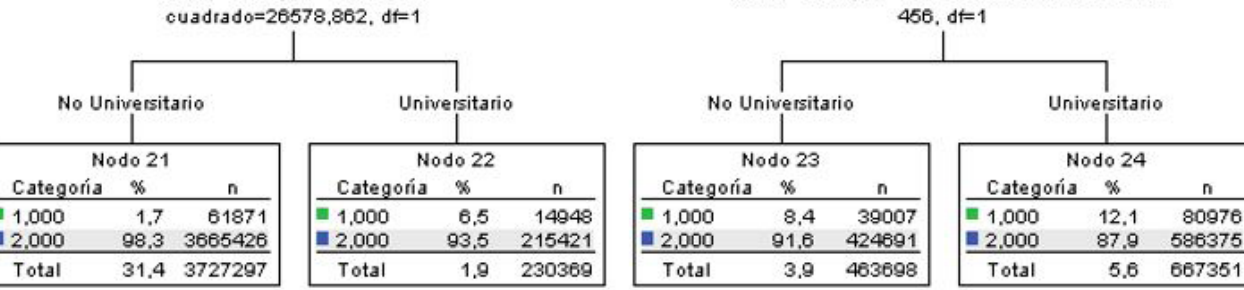

Fuente: Elaboración propia a partir de los microdatos del censo de la población española 2011. INE

ANEXO 1.2

ANÁLISIS ÁRBOL. HOGARES UNIPERSONALES ADULTOS 35-64 AÑOS

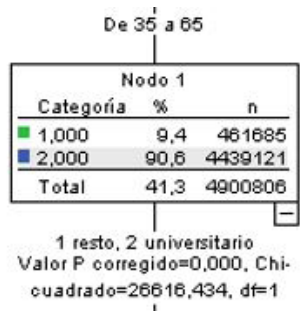

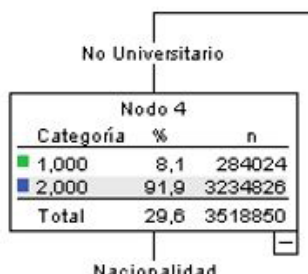

Nacionalida

Valor $P$ corregido=0,000, cuadrado=15773,239, $d t=1$

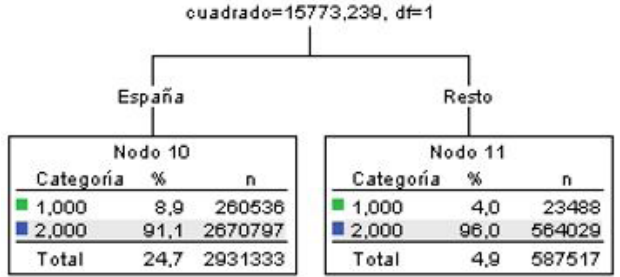

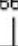

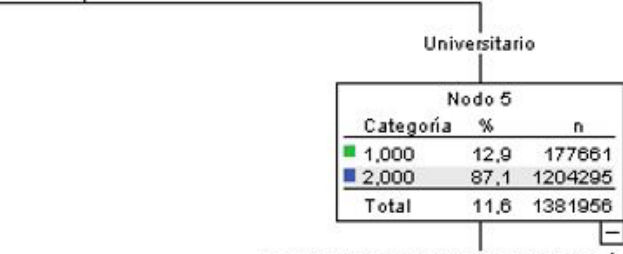

1. menos de $5000 ; 2$ de 2 a 100; 3 más de 100 Valor P oorregido=0,000. Chi-cuadrad $0=0277$

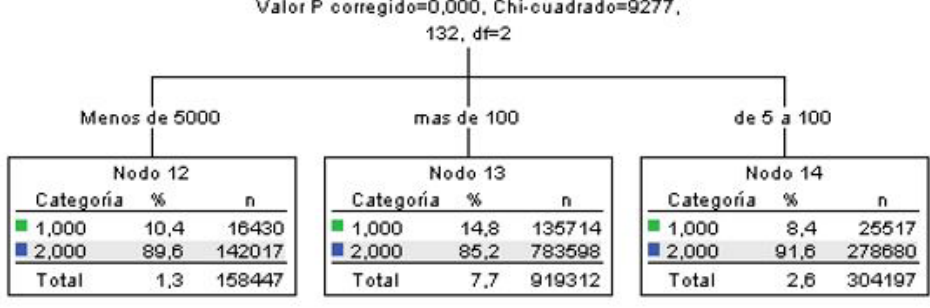

Fuente: Elaboracion propia a partir de los microdatos del censo de la población española 2011. INE 
ANEXO 1.3

ANÁLISIS ÁRBOL. HOGARES UNIPERSONALES MAYORES. >65 AÑOS

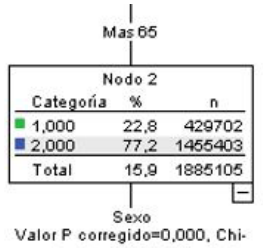

cuadrado $=90881,493, d f=1$
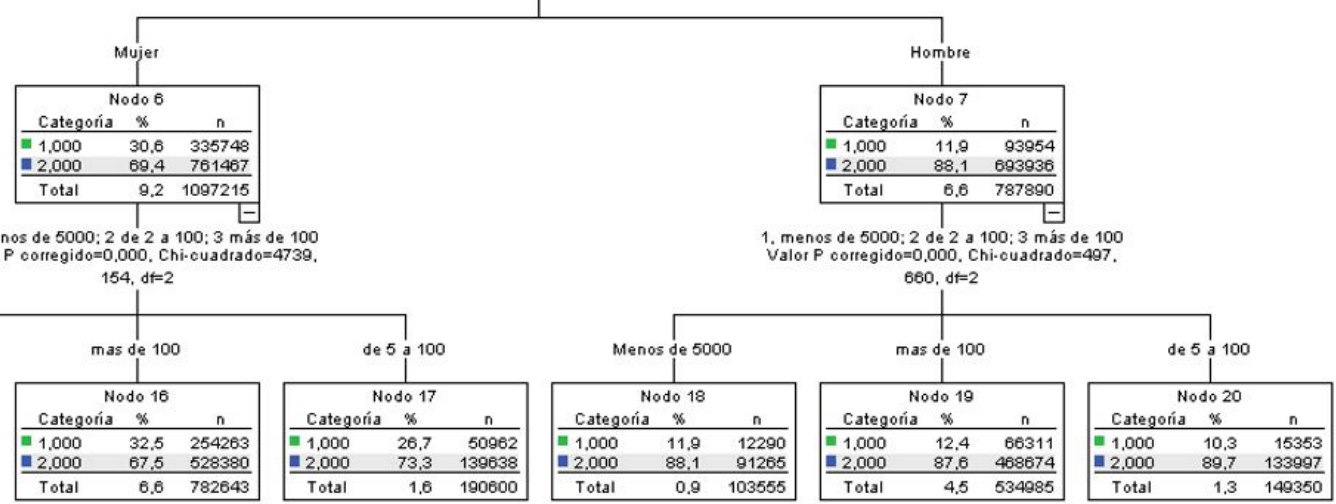

Fuente: Elaboración propia a partir de los microdatos del censo de la población española 2011. INE 